\title{
Multi-attribute decision making parametric optimization in two-stage hot cascade vortex tube through grey relational analysis
}

\author{
Racharla Madhu Kumar ${ }^{1, *}$, NVVS Sudheer ${ }^{2}$, and Katam Ganesh Babu ${ }^{3}$ \\ ${ }^{1}$ Research Scholar, ANU, School of Mechanical Engineering, R.G.M. College of Engineering \& Technology, Nandyal, India \\ 2 Department of Mechanical Engineering, RVR \& JC College of Engineering, Guntur, Andhra Pradesh, India \\ ${ }^{3}$ Department of Mechanical Engineering, NIT Andhra, Tadepalligudem, Andhra Pradesh, India
}

Received: 15 July 2019 / Accepted: 26 August 2020

\begin{abstract}
By setting two vortex tubes in hot cascade type Vortex tube manner, can achieve two cooling points for spot cooling applications with the single input. These cooling points play a vital role to cool tools in machining operations. The present work aims to optimize the output parameters such as outlet temperature, Coefficient of Performance (COP). Based on the literature, the performance of this vortex tube mainly depends on its input parameters such as air inlet pressure, length to diameter ratio, and the number of nozzles. In the present work, the above input parameters have been experimented on this vortex tube, based on the Taguchi L18 array. The optimal condition for both temperatures, COP at hot and cold outlets was calculated using grey relational analysis (GRA). The obtained experimental results were analyzed using the ANOVA approach. Also for multi responses, 1st and 2nd order predicted mathematical models developed by using Minitab 18 software and its accuracy checked. The achieved results are at first spot cooling point temperature $294.9 \mathrm{~K}, \mathrm{COP}_{c 1}$ as 0.0203 , second spot cooling point temperature $284.2 \mathrm{~K}$, and $\mathrm{COP}_{c 2}$ as 0.1628 . This work proved that for solving multiattribute decision-making problems, grey relational analysis methodology was efficient.
\end{abstract}

Keywords: Coefficient of Performance / experiment / grey relational analysis / Taguchi / temperature difference / vortex tube

\section{Introduction}

The pressurized gas/air as an input for the vortex tube and this was split into two streams; one is towards the cold outlet and another one as a hot outlet. In this work, the two vortex tubes are arranged as a hot cascade type vortex tube as shown in Figure 1. The design parameters are playing a vital role in the proper understanding of the heat transfer phenomena of the vortex tube. Wua et al. [1] taken a new intake flow passage and nozzle design then vortex tube performance improved. By using artificial neural networks, Dincer et al. [2] investigated the performance of the vortex tube by varying the $\mathrm{L} / \mathrm{D}$ ratio and nozzle number. In this investigation, MATLAB 6.5 NN toolbox software was used and compared by making use of independent statistical t-test in SPSS.

Pinar et al. [3], succeeded to improve the temperature difference between hot, cold ends of the Vortex Tubes and statistically proved that the air pressure at the inlet, L/D ratio, and nozzles are significant factors in this study.

\footnotetext{
* e-mal: madhukumar325@gmail.com
}

Aydınet al. [4] was introduced a new geometry as a 'Helical swirl flow generator' at the cold end side. In this investigation, they found the performance of the vortex tube by varying the helical length of the swirl flow generator. Dincer [5] found the performance of a three-fold and six-cascade type vortex tube. In this experiment, $\Delta \mathrm{T}$ maximum value is $67.6^{\circ} \mathrm{C}$ at six vortex tubes third situation. By using Response Surface Methodology (RSM), Prabakaran et al. [6] have proved that the diameter of the nozzle, inlet air pressure are the most influencing factors for the cold end side temperature difference and performance of the vortex tube. Berber et al. [7] used a Rule-Based Mamdani-Type Fuzzy modeling technique for modeled and investigated experimentally heating and cooling performance of the vortex tube. With different geometric constructions of vortex tube, analyzed heating and cooling performances based on actual values. By using response surface methodology (RSM), Bovand et al. [8] optimized the standard Ranque-Hilsch vortex tube for maximum cold air temperature difference. In this study, they found that the optimum value of cold orifice diameter $\left(d_{c}\right)$ to the tube diameter $(D)$ is 0.5 . Jayaraman et al. [9] have evidenced that the grey relational analysis was 


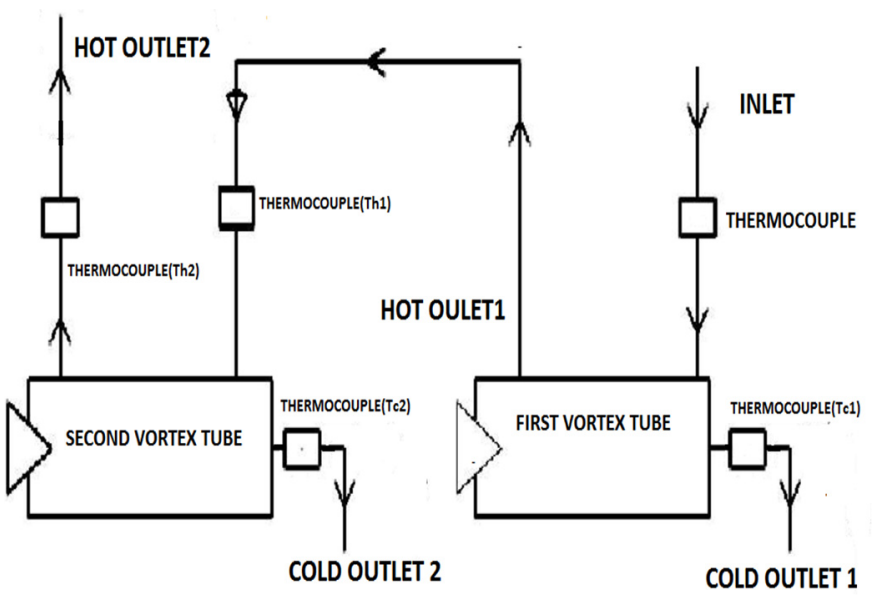

Fig. 1. Schematic diagram of the hot cascade type two-stage vortex tube.

an efficient method for the application of multi-attribute decision making parametric optimization. Venkata Sivareddy et al. [10] were studied the effect of cutting and vibrating parameters in a VAT on cutting force, cutting temperature, equivalent stress, and compressive maximum circumferential residual stress (MCRS). In this investigation, Taguchi based analysis of variance (ANOVA) and grey relational analysis (GRA) methods were used to optimize VAT of Ti6Al4V alloy parameters. Nguyen et al. [11] were studied to optimize the conducted parameter sets for 3D fused deposition modeling (FDM) products. The parameter sets were layer height, infill percentage, printing temperature, printing speed with different levels were experimented and analyzed to build mathematic models.

Hawary et al. [12] were investigated, by using a multiobjective genetic algorithm (MOGA) to optimize the power split between an internal combustion engine and a hydraulic motor to improve fuel economy. The conclusion of this investigation was improvement achieved by the $\mathrm{HHV}$ in terms of fuel economy is recorded as much as $5.55 \%$ for highway and $6.5 \%$ for city drive cycles. So many scientists conducted experiments, analyzed, and theoretically studied on this device. Some of the scientists are K.G. Hellyar [13], N. Logothetis et al. [14], J.L. Deng [15], G. Taguchi [16], G.S. Peace [17], P.J. Ross [18], K. Dincer [19], A.M. Pinar [20], S. Eiamsa-ard [21], M. Bovand $[22,23]$, V. Kirmaci [24]. In this experimental investigation the input parameters were taken as inlet pressure of air, length $(L)$ /diameter $(D)$ ratio, nozzles number to optimize the temperature and coefficient of performance (COP) at hot outlets and cold outlets through grey relational analysis. Also for multi responses, 1st and 2nd order predicted mathematical models developed by using Minitab 18 software and its accuracy checked.

\section{Experimental details}

The schematic representation and photo snap of the vortex tube experimental setup as shown in Figures 1 and 2. The specifications and geometric dimensions are as follows. $6.75,9$, and $13.5 \mathrm{~mm}$ are the inner diameters $(D)$ of the

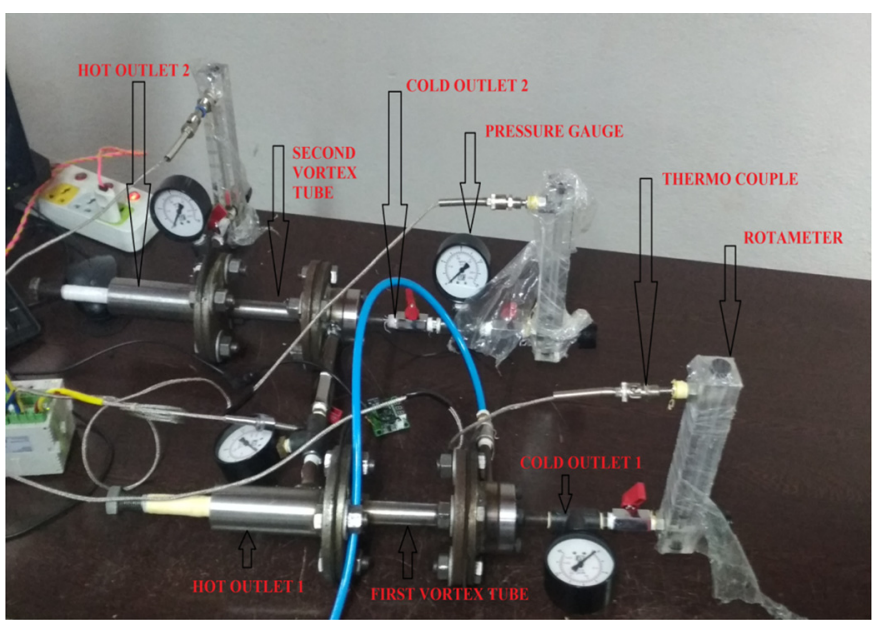

Fig. 2. Photo snap of the hot cascade type two-stage vortex tube.

vortex tube, 10,15 , and 20 are $L / D$ ratio 4,5 , and 6 are the number of nozzles $(N)$. In this test, the pressurized air is given as input, conical tip plug used as an obstruction of the vortex tube at the hot end which an angle of $45^{\circ}$. Inlet air pressure was taken as 7, 8, 9, 10, 11, and 12 bar. With Fe-K thermocouples, the temperatures of air at different locations measured. By using Taguchi L18 array experiments were planned as per taking three factors (input parameters) such as pressure $(P)$ at six levels, $L / D$ ratio, and nozzles $(N)$ at three levels. The level of pressure varies from the 7-12 bar as levels of 1-6, respectively. The level of $L / D$ ratio varies from 10 as level 1,15 as level 2 , and 20 as level 3, respectively. The level of the number of nozzles varies from 4 as level 1, 5 as level 2 , and 6 as level 3 respectively. Taguchi L18 array design constitutes a total of 18 experimental runs. The experimental results of both temperatures and COP's are shown in Table 1.

\section{Grey relational analysis optimization methodology}

The problem in which multi-responses as output parameters. By using grey relational analyses to optimize these output parameters. With the help of grey relational grade in grey relational analyses optimize multi responses. In the optimization problem, multi responses are converted into a single relational grade.

Step 1

First, normalize the given experimental data because parameters have different units.

$$
A *_{x}(i)=\frac{\max A x(i)-A x(i)}{\max A x(i)-\min A x(i)}
$$

where $x=1, \ldots, p ; i=1, \ldots, q$; the number of experimental data is $p$, and the number of responses is $q$. The original sequence as $A_{x}(i)$, data preprocessing after sequencing as $A^{*}{ }_{x}(i)$, the maximum value of $A_{x}(i)$ as $\max A_{x}(i)$, the minimum value of $A x(i)$ as min $A x(i)$, and $A$ is the desired value. 
Table 1. Experimental results at hot and cold outlets as per Taguchi L18 array.

\begin{tabular}{|c|c|c|c|c|c|c|c|c|c|c|c|}
\hline \multirow[t]{2}{*}{ Run. No } & \multicolumn{3}{|c|}{ Input Parameters } & \multicolumn{8}{|c|}{ Experimental results } \\
\hline & $P$ & $L / D$ & $N$ & $T_{h 1}(\mathrm{~K})$ & $\mathrm{T}_{c 1}(\mathrm{~K})$ & $\mathrm{T}_{h 2}(\mathrm{~K})$ & $\mathrm{T}_{c 2}(\mathrm{~K})$ & $\mathrm{COP}_{h 1}$ & $\mathrm{COP}_{c 1}$ & $\mathrm{COP}_{h 2}$ & $\mathrm{COP}_{c 2}$ \\
\hline 1 & 7 & 10 & 4 & 304.8 & 300.7 & 321.2 & 290.7 & 0.0065 & 0.0057 & 0.0164 & 0.0975 \\
\hline 2 & 7 & 15 & 5 & 306.0 & 300.0 & 322.5 & 290.5 & 0.0102 & 0.0078 & 0.0148 & 0.1064 \\
\hline 3 & 7 & 20 & 6 & 305.1 & 300.8 & 321.0 & 291.8 & 0.0075 & 0.0053 & 0.0118 & 0.0933 \\
\hline 4 & 8 & 10 & 4 & 305.5 & 299.8 & 322.0 & 291.8 & 0.0075 & 0.0084 & 0.0179 & 0.0916 \\
\hline 5 & 8 & 15 & 5 & 307.0 & 299.0 & 323.2 & 290.0 & 0.0114 & 0.0109 & 0.0154 & 0.1114 \\
\hline 6 & 8 & 20 & 6 & 307.0 & 299.0 & 322.5 & 291.0 & 0.0120 & 0.0103 & 0.0115 & 0.1099 \\
\hline 7 & 9 & 10 & 5 & 306.8 & 298.4 & 323.5 & 290.5 & 0.0104 & 0.0117 & 0.0149 & 0.1029 \\
\hline 8 & 9 & 15 & 6 & 307.6 & 298.2 & 323.7 & 289.2 & 0.0122 & 0.0125 & 0.0157 & 0.1143 \\
\hline 9 & 9 & 20 & 4 & 308.2 & 297.1 & 324.1 & 289.4 & 0.0138 & 0.0154 & 0.0164 & 0.1254 \\
\hline 10 & 10 & 10 & 6 & 308.2 & 297.0 & 323.6 & 288.5 & 0.0127 & 0.0154 & 0.0105 & 0.1227 \\
\hline 11 & 10 & 15 & 5 & 310.5 & 294.9 & 326.6 & 284.2 & 0.0188 & 0.0203 & 0.0150 & 0.1628 \\
\hline 12 & 10 & 20 & 4 & 309.8 & 295.5 & 326.5 & 288.1 & 0.0171 & 0.0187 & 0.0159 & 0.1385 \\
\hline 13 & 11 & 10 & 5 & 308.5 & 295.9 & 325.7 & 288.1 & 0.0129 & 0.0174 & 0.0152 & 0.1213 \\
\hline 14 & 11 & 15 & 6 & 309.9 & 295.7 & 325.5 & 286.2 & 0.0161 & 0.0181 & 0.0139 & 0.1419 \\
\hline 15 & 11 & 20 & 4 & 310.0 & 295.2 & 326.8 & 287.9 & 0.0168 & 0.0188 & 0.0165 & 0.1380 \\
\hline 16 & 12 & 10 & 6 & 307.8 & 297.3 & 322.9 & 288.4 & 0.0102 & 0.0142 & 0.0101 & 0.1205 \\
\hline 17 & 12 & 15 & 4 & 309.5 & 295.7 & 325.7 & 287.3 & 0.0146 & 0.0174 & 0.0156 & 0.1332 \\
\hline 18 & 12 & 20 & 5 & 310.2 & 295.9 & 327.1 & 285.1 & 0.0161 & 0.0172 & 0.0143 & 0.1491 \\
\hline
\end{tabular}

Step 2

Grey relational coefficient $g_{x}$ (i) calculated by using below formula from normalized data

$$
g_{x}(i)=\frac{\Delta \min +\xi \Delta \max }{\Delta 0 x(i)+\xi \Delta \max }
$$

where the comparability sequence and a reference sequence is the deviation sequence $\Delta_{0 i}$ and

$$
\Delta_{0 x}=A_{0}(i)-A_{x}(i)
$$

where $A_{0}(i), A_{x}(i)$ are the reference, comparability sequences. The minimum, maximum values of the absolute differences $\left(\Delta_{0 x}\right)$ are $\Delta_{\min }, \Delta_{\max }$. The identification coefficient $(\xi)$ range between 0 to 1 and take value in this work was 0.5 .

Step-3

Below formula used to calculate Grey relational grade (GRG)

$$
\gamma_{i}=\frac{1}{n} \sum_{k=1}^{n} \xi(k)
$$

where $i$ th experiment GRG as $\gamma_{i}$ for every response. Using grey relational analysis, multi responses are converted into a single response optimization problem. The highest average grade value is chosen in the mean response table, and this is an optimal parametric combination for multiresponses.

\section{Implementation of grey relational analysis optimization methodology}

Step 1

First, normalize the experimental data like temperatures and COP's using equation (1) and values are tabulated shown in Table 2.

Step 2

From Table $2, g_{x}(i)$ was calculated using equation (2) and $\xi$ is taken as 0.5 . The calculated values are tabulated shown in Table 3.

Step 3

By using equation (3), grey relational grade (GRG) was calculated using grey relational coefficients. The calculated GRG values are tabulated in Table 3 . Here multi responses converted into a single response.

\section{Results and discussion}

Each input parameter effects at different levels are plotted (Fig. 3) based on GRG values. The highest average grade value is chosen in mean Table 4 , and this is an optimal parametric combination for multi-responses. Thus, the optimal settings for multi-responses become P4-L/D2-N2, i.e., the pressure of $10 \mathrm{bar}, \mathrm{L} / \mathrm{D}$ ratio of 15 , and Nozzles of 5 . The mean grey relational grade (Fig. 3) higher values represent the maximum value of temperature at hot outlets, $\mathrm{COP}$ at hot and cold outlets, and minimum value of temperature at cold outlets. Based in Table 4, the air 
Table 2. Generation values of grey relational analysis.

\begin{tabular}{|c|c|c|c|c|c|c|c|c|c|}
\hline Run. No & & $T_{h 1}$ & $T_{c 1}$ & $T_{h 2}$ & $T_{c 2}$ & $\mathrm{COP}_{h 1}$ & $\mathrm{COP}_{c 1}$ & $\mathrm{COP}_{h 2}$ & $\mathrm{COP}_{c 2}$ \\
\hline 1 & 1.000 & & 0.017 & 0.967 & 0.145 & 1.0000 & 0.9737 & 0.1873 & 0.9172 \\
\hline 2 & 0.789 & & 0.136 & 0.754 & 0.171 & 0.7038 & 0.8363 & 0.4011 & 0.7922 \\
\hline 3 & 0.947 & & 0.000 & 1.000 & 0.000 & 0.9195 & 1.0000 & 0.7775 & 0.9758 \\
\hline 4 & 0.877 & & 0.169 & 0.836 & 0.000 & 0.9175 & 0.7978 & 0.0000 & 1.0000 \\
\hline 5 & 0.614 & & 0.305 & 0.639 & 0.237 & 0.6018 & 0.6304 & 0.3237 & 0.7211 \\
\hline 6 & 0.614 & & 0.305 & 0.754 & 0.105 & 0.5562 & 0.6673 & 0.8218 & 0.7421 \\
\hline 7 & 0.649 & & 0.407 & 0.590 & 0.171 & 0.6815 & 0.5766 & 0.3853 & 0.8401 \\
\hline 8 & 0.509 & & 0.441 & 0.557 & 0.342 & 0.5353 & 0.5206 & 0.2849 & 0.6802 \\
\hline 9 & 0.404 & & 0.627 & 0.492 & 0.316 & 0.4059 & 0.3289 & 0.1896 & 0.5254 \\
\hline 10 & 0.404 & & 0.644 & 0.574 & 0.434 & 0.4941 & 0.3275 & 0.9459 & 0.5628 \\
\hline 11 & 0.000 & & 1.000 & 0.082 & 1.000 & 0.0000 & 0.0000 & 0.3709 & 0.0000 \\
\hline 12 & 0.123 & & 0.898 & 0.098 & 0.487 & 0.1379 & 0.1050 & 0.2584 & 0.3410 \\
\hline 13 & 0.351 & & 0.831 & 0.230 & 0.487 & 0.4785 & 0.1962 & 0.3474 & 0.5829 \\
\hline 14 & 0.105 & & 0.864 & 0.262 & 0.737 & 0.2219 & 0.1490 & 0.5168 & 0.2936 \\
\hline 15 & 0.088 & & 0.949 & 0.049 & 0.513 & 0.1645 & 0.1017 & 0.1837 & 0.3481 \\
\hline 16 & 0.474 & & 0.593 & 0.689 & 0.447 & 0.7018 & 0.4052 & 1.0000 & 0.5940 \\
\hline 17 & 0.175 & & 0.864 & 0.230 & 0.592 & 0.3408 & 0.1942 & 0.2904 & 0.4148 \\
\hline 18 & 0.053 & & 0.831 & 0.000 & 0.882 & 0.2251 & 0.2091 & 0.4565 & 0.1928 \\
\hline
\end{tabular}

Table 3. Grey relational coefficient and grey relational grade value.

\begin{tabular}{|c|c|c|c|c|c|c|c|c|c|c|}
\hline \multirow[t]{2}{*}{ Run. No } & \multicolumn{8}{|c|}{ Grey relational coefficient } & \multirow[t]{2}{*}{ GRG } & \multirow[t]{2}{*}{ RANK } \\
\hline & $T_{h 1}$ & $\mathrm{~T}_{c 1}$ & $\mathrm{~T}_{h 2}$ & $\mathrm{~T}_{c 2}$ & $\mathrm{COP}_{h 1}$ & $\mathrm{COP}_{c 1}$ & $\mathrm{COP}_{h 2}$ & $\mathrm{COP}_{c 2}$ & & \\
\hline 1 & 0.333 & 0.337 & 0.341 & 0.369 & 0.333 & 0.339 & 0.728 & 0.353 & 0.392 & 17 \\
\hline 2 & 0.388 & 0.366 & 0.399 & 0.376 & 0.415 & 0.374 & 0.555 & 0.387 & 0.408 & 16 \\
\hline 3 & 0.345 & 0.333 & 0.333 & 0.333 & 0.352 & 0.333 & 0.391 & 0.339 & 0.345 & 18 \\
\hline 4 & 0.363 & 0.376 & 0.374 & 0.333 & 0.353 & 0.385 & 1.000 & 0.333 & 0.440 & 14 \\
\hline 5 & 0.449 & 0.418 & 0.439 & 0.396 & 0.454 & 0.442 & 0.607 & 0.409 & 0.452 & 12 \\
\hline 6 & 0.449 & 0.418 & 0.399 & 0.358 & 0.473 & 0.428 & 0.378 & 0.403 & 0.413 & 15 \\
\hline 7 & 0.435 & 0.457 & 0.459 & 0.376 & 0.423 & 0.464 & 0.565 & 0.373 & 0.444 & 13 \\
\hline 8 & 0.496 & 0.472 & 0.473 & 0.432 & 0.483 & 0.490 & 0.637 & 0.424 & 0.488 & 10 \\
\hline 9 & 0.553 & 0.573 & 0.504 & 0.422 & 0.552 & 0.603 & 0.725 & 0.488 & 0.553 & 8 \\
\hline 10 & 0.553 & 0.584 & 0.466 & 0.469 & 0.503 & 0.604 & 0.346 & 0.470 & 0.499 & 9 \\
\hline 11 & 1.000 & 1.000 & 0.859 & 1.000 & 1.000 & 1.000 & 0.574 & 1.000 & 0.929 & 1 \\
\hline 12 & 0.803 & 0.831 & 0.836 & 0.494 & 0.784 & 0.826 & 0.659 & 0.595 & 0.728 & 4 \\
\hline 13 & 0.588 & 0.747 & 0.685 & 0.494 & 0.511 & 0.718 & 0.590 & 0.462 & 0.599 & 7 \\
\hline 14 & 0.826 & 0.787 & 0.656 & 0.655 & 0.693 & 0.770 & 0.492 & 0.630 & 0.689 & 5 \\
\hline 15 & 0.851 & 0.908 & 0.910 & 0.507 & 0.752 & 0.831 & 0.731 & 0.590 & 0.760 & 3 \\
\hline 16 & 0.514 & 0.551 & 0.421 & 0.475 & 0.416 & 0.552 & 0.333 & 0.457 & 0.465 & 11 \\
\hline 17 & 0.740 & 0.787 & 0.685 & 0.551 & 0.595 & 0.720 & 0.633 & 0.547 & 0.657 & 6 \\
\hline 18 & 0.905 & 0.747 & 1.000 & 0.809 & 0.690 & 0.705 & 0.523 & 0.722 & 0.762 & 2 \\
\hline
\end{tabular}

inlet pressure has the utmost inducing effect than the $\mathrm{L} / \mathrm{D}$ ratio and the number of nozzles. The sequence of the importance of input parameters on multi-responses is pressure $>\mathrm{L} / \mathrm{D}$ ratio $>$ number of nozzles.
Based on grey relational grade value analysis of variance (ANOVA) table is formulated as shown in Table 5. In this ANOVA method, which parameter $\mathrm{P}$-value is less than 0.05 that parameter is a significant 
parameter. In this ANOVA table, pressure and $\mathrm{L} / \mathrm{D}$ ratio parameters, $\mathrm{P}$-values are 0.003 and 0.034 , respectively. These values are less than 0.05 at $95 \%$ confidence level. So, pressure and $\mathrm{L} / \mathrm{D}$ ratio are the significant and number of nozzles insignificant input parameters.

The multiple regression model developed by using Minitab 18 software for all responses such as the temperature at hot outlet $1\left(T_{h 1}\right)$, temperature at cold outlet $1\left(\mathrm{~T}_{c 1}\right)$, the temperature at hot outlet $2\left(\mathrm{~T}_{h 2}\right)$, the temperature at cold outlet $2\left(\mathrm{~T}_{c 2}\right), \mathrm{COP}$ of hot outlet 1 $\left(\mathrm{COP}_{h 1}\right), \mathrm{COP}$ of cold outlet $1\left(\mathrm{COP}_{c 1}\right), \mathrm{COP}$ of hot outlet 2 $\left(\mathrm{COP}_{h 2}\right)$, and $\mathrm{COP}$ of cold outlet $2\left(\mathrm{COP}_{c 2}\right)$ at $95 \%$ confidence level considering pressure (P), Length (L) /Diameter (D) ratio and the number of nozzles $(\mathrm{N})$ as input parameters. Based on obtained results, 1st and 2nd order mathematical models for the temperature at hot outlet 1 $\left(T_{h 1}\right)$, Temperature at cold outlet $1\left(\mathrm{~T}_{c 1}\right)$, Temperature at

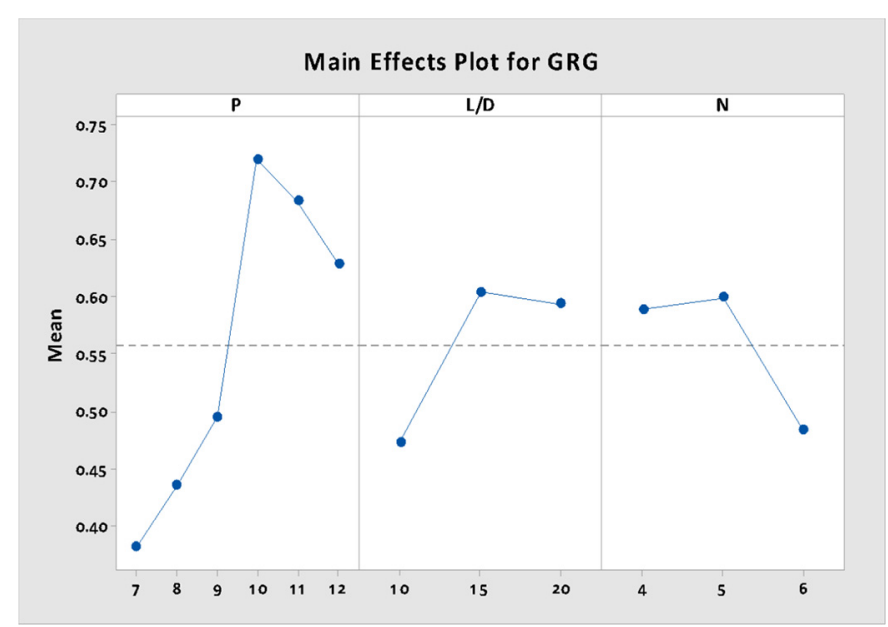

Fig. 3. Main effect plot of grey relational grade. hot outlet $2\left(\mathrm{~T}_{h 2}\right)$, Temperature at cold outlet $2\left(\mathrm{~T}_{c 2}\right)$, COP of hot outlet $1\left(\mathrm{COP}_{h 1}\right), \mathrm{COP}$ of cold outlet $1\left(\mathrm{COP}_{c 1}\right), \mathrm{COP}$ of hot outlet $2\left(\mathrm{COP}_{h 2}\right)$, and $\mathrm{COP}$ of cold outlet $2\left(\mathrm{COP}_{c 2}\right)$ are presented in equations (4)-(19) along with their $R^{2}$ value respectively.

The determination coefficient values of 1 st order regression model show very less compare to the 2 nd order regression model particularly for the temperature at cold outlet 2 , which is $63.1 \%$, and 2 nd order model $R^{2}$ value is close to 1 . The high $R^{2}$ value model represents the best fit of the model, i.e., the 2 nd order model is the best fit of the model. The 2nd order model predicted values and experimental values comparison shown in Figures 4-11. The correlations for predicted and experimental results were best in the 2 nd order model. In this experiment, the 2nd order model gives sufficient predicts the expected results compared 1st order model.

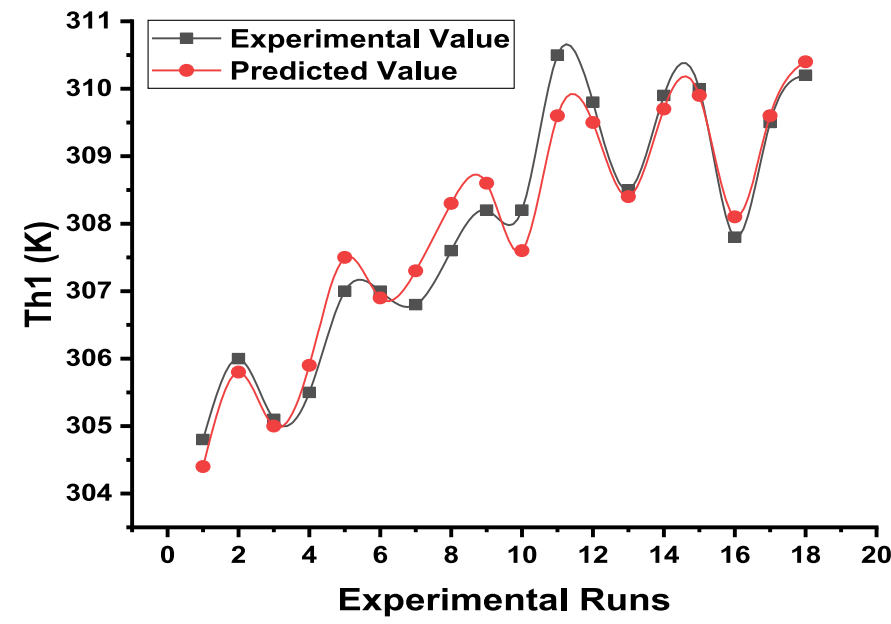

Fig. 4. Experimental vs. predicted values of temperature at hot outlet $1\left(T_{h 1}\right)$.

Table 4. Effects on mean grey relational grade.

\begin{tabular}{lllllllll}
\hline Factors & \multicolumn{9}{c}{ Mean grey relational grade } & \multirow{2}{*}{ Max-Min } & Rank \\
\cline { 2 - 7 } & Level 1 & Level 2 & Level 3 & Level 4 & Level 5 & Level 6 & & \\
\hline$P$ & 0.382 & 0.435 & 0.495 & 0.719 & 0.683 & 0.628 & 0.337 & 1 \\
$L / D$ & 0.473 & 0.604 & 0.594 & & & & 0.131 & 2 \\
$N$ & 0.588 & 0.599 & 0.483 & & & & 0.116 & 3 \\
\hline
\end{tabular}

Table 5. ANOVA results on grey relational grade.

\begin{tabular}{lrrrrrll}
\hline Source & $D F$ & Adj SS & Adj MS & $F$ & $P$ & Remarks & $\begin{array}{c}\text { Contribution } \\
\text { ratio }(\%)\end{array}$ \\
\hline$P$ & 5 & 0.2899 & 0.0580 & 9.76 & 0.003 & Significant & 64.45 \\
$L / D$ & 2 & 0.0631 & 0.0316 & 5.31 & 0.034 & Significant & 14.02 \\
$N$ & 2 & 0.0489 & 0.0244 & 4.11 & 0.059 & Insignificant & 10.87 \\
Error & 8 & 0.0475 & 0.0059 & & & & \\
Total & 17 & 0.4498 & & & & & \\
\hline
\end{tabular}




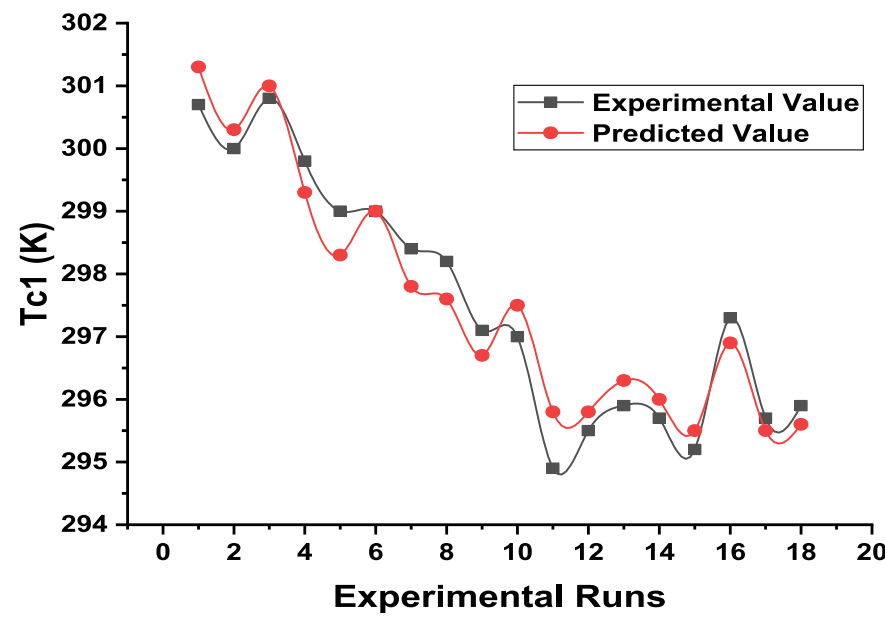

Fig. 5. Experimental vs. predicted values of temperature at cold outlet $1\left(\mathrm{~T}_{c 1}\right)$.

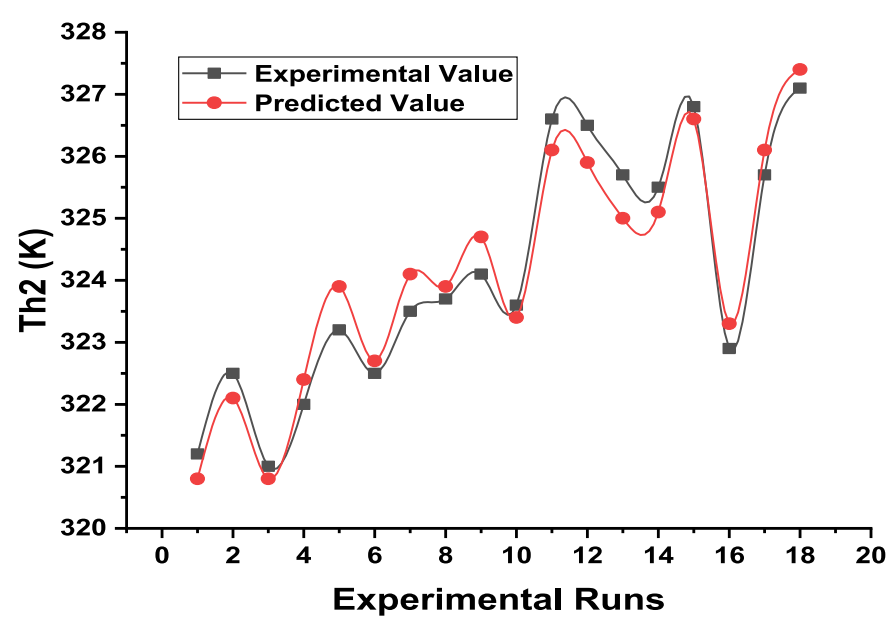

Fig. 6. Experimental vs. predicted values of temperature at hot outlet $2\left(\mathrm{~T}_{h 2}\right)$.

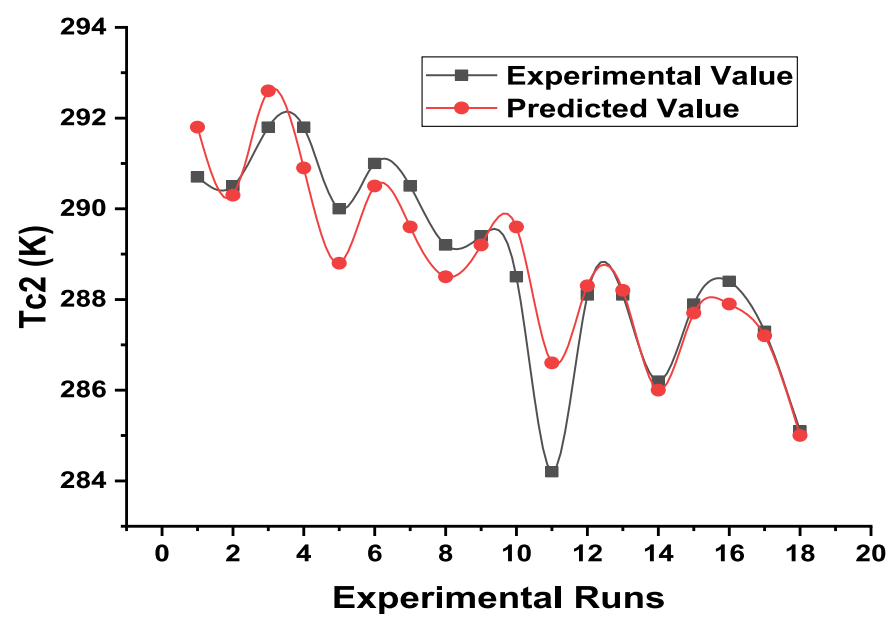

Fig. 7. Experimental vs. predicted values of temperature at cold outlet $2\left(\mathrm{~T}_{c 2}\right)$.

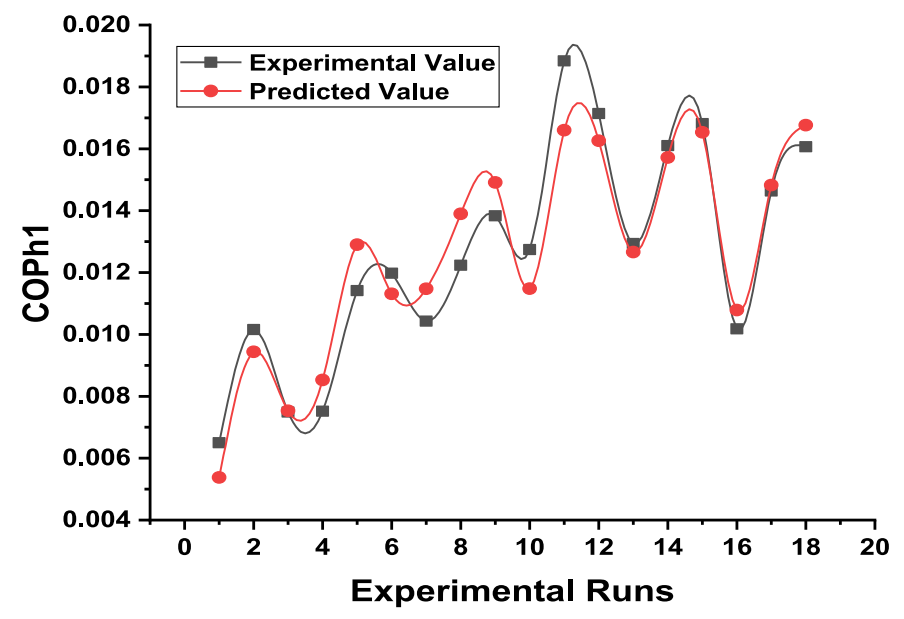

Fig. 8. Experimental vs. predicted values of coefficient of performance at hot outlet $1\left(\mathrm{COP}_{h 1}\right)$.

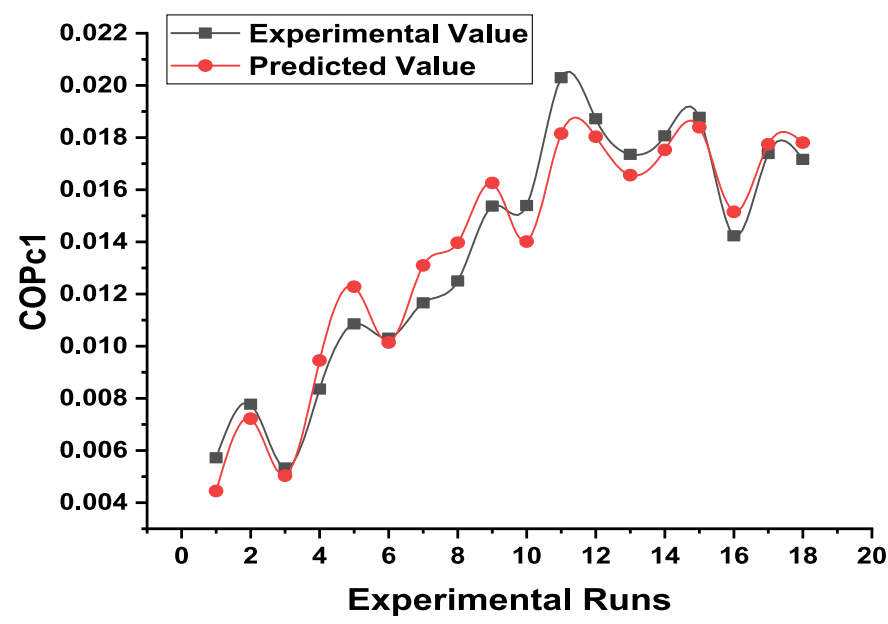

Fig. 9. Experimental vs. predicted values of coefficient of performance at cold outlet $1\left(\mathrm{COP}_{c 1}\right)$.

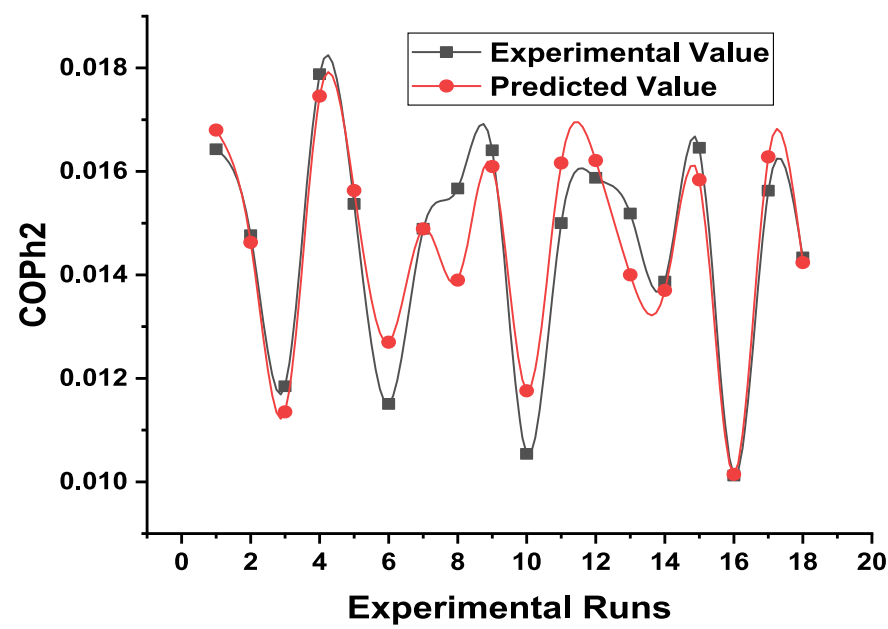

Fig. 10. Experimental vs. predicted values of coefficient of performance at hot outlet $2\left(\mathrm{COP}_{h 2}\right)$. 


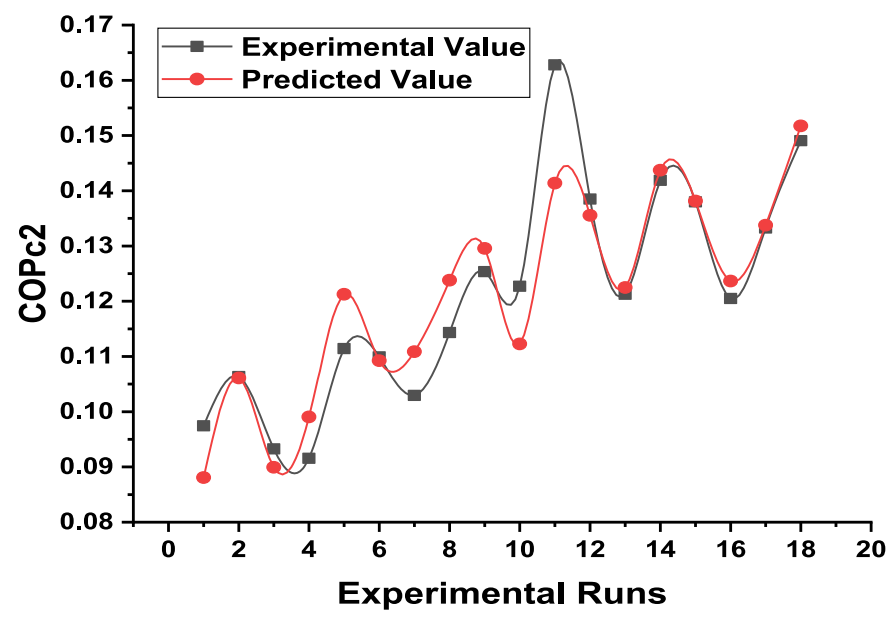

Fig. 11. Experimental vs. predicted values of coefficient of performance at cold outlet $2\left(\mathrm{COP}_{c 2}\right)$.

\subsection{First-order model}

$$
\begin{aligned}
\mathrm{T}_{\mathrm{h} 1} & =298.19+0.863 \mathrm{P}+0.1429 \mathrm{~L} / \mathrm{D}-0.124 \mathrm{~N} \\
\mathrm{R}^{2} & =80.1 \% \\
\mathrm{~T}_{\mathrm{c} 1} & =306.66-0.974 \mathrm{P}-0.0884 \mathrm{~L} / \mathrm{D}+0.297 \mathrm{~N} \\
\mathrm{R}^{2} & =80.4 \% \\
\mathrm{~T}_{\mathrm{h} 2} & =316.38+0.87 \mathrm{P}+0.1428 \mathrm{~L} / \mathrm{D}-0.532 \mathrm{~N} \\
\mathrm{R}^{2} & =75.8 \% \\
\mathrm{~T}_{\mathrm{c} 2} & =299.36-0.963 \mathrm{P}-0.079 \mathrm{~L} / \mathrm{D}-0.041 \mathrm{~N} \\
\mathrm{R}^{2} & =63.1 \%
\end{aligned}
$$

$$
\begin{aligned}
& \mathrm{COP}_{\mathrm{h} 1}=-0.0042+0.00134 \mathrm{P}+0.000378 \mathrm{~L} / \mathrm{D}-0.000318 \mathrm{~N} \\
& \mathrm{R}^{2}=64.9 \% \\
& \mathrm{COP}_{\mathrm{c} 1=}-0.00794+0.002273 \mathrm{P}+0.000205 \mathrm{~L} / \mathrm{D} \\
& -0.000623 \mathrm{~N} \quad \mathrm{R}^{2}=76.1 \% \\
& \mathrm{COP}_{\mathrm{h} 2}=0.02685-0.000172 \mathrm{P}-0.000012 \mathrm{~L} / \mathrm{D} \\
& -0.002099 \mathrm{~N} \quad \mathrm{R}^{2}=67.7 \% \\
& \mathrm{COP}_{\mathrm{c} 2}= \\
& 0.0235+0.00833 \mathrm{P}+0.00161 \mathrm{~L} / \mathrm{D} \\
& -0.00112 \mathrm{~N} \quad \mathrm{R}^{2}=66.2 \% .
\end{aligned}
$$

\subsection{Second order model}

$$
\begin{aligned}
\mathrm{T}_{\mathrm{h} 1}= & 270.4+4.12 \mathrm{P}+0.873 \mathrm{~L} / \mathrm{D}+3.06 \mathrm{~N}-0.2074 \mathrm{P}^{*} \mathrm{P} \\
& -0.0286 \mathrm{~L} / \mathrm{D}^{*} \mathrm{~L} / \mathrm{D}-0.38 \mathrm{~N}^{*} \mathrm{~N}+0.0188 \mathrm{P}^{*} \mathrm{~L} / \mathrm{D} \\
& +0.08 \mathrm{P}^{*} \mathrm{~N}-0.0058 \mathrm{~L} / \mathrm{D}^{*} \mathrm{~N} \quad \mathrm{R}^{2}=95 \%
\end{aligned}
$$

$$
\begin{aligned}
\mathrm{T}_{\mathrm{c} 1}= & 340.7-5.75 \mathrm{P}-0.827 \mathrm{~L} / \mathrm{D}-2.63 \mathrm{~N} \\
& +0.2547 \mathrm{P}^{*} \mathrm{P}+0.0199 \mathrm{~L} / \mathrm{D}^{*} \mathrm{~L} / \mathrm{D}+0.343 \mathrm{~N}^{*} \mathrm{~N} \\
& +0.0199 \mathrm{P}^{*} \mathrm{~L} / \mathrm{D}-0.048 \mathrm{P}^{*} \mathrm{~N}+0.0027 \mathrm{~L} / \mathrm{D}^{*} \mathrm{~N} \\
\mathrm{R}^{2}= & 94 \% \\
\mathrm{~T}_{\mathrm{h} 2}= & 273.4+4.64 \mathrm{P}+0.212 \mathrm{~L} / \mathrm{D}+9.69 \mathrm{~N} \\
& -0.2177 \mathrm{P}^{*} \mathrm{P}-0.0177 \mathrm{~L} / \mathrm{D}^{*} \mathrm{~L} / \mathrm{D}-0.967 \mathrm{~N}^{*} \mathrm{~N} \\
& +0.0439 \mathrm{P}^{*} \mathrm{~L} / \mathrm{D}-0.055 \mathrm{P}^{*} \mathrm{~N}+0.0109 \mathrm{~L} / \mathrm{D}^{*} \mathrm{~N} \\
\mathrm{R}^{2}= & 94.6 \% \\
\mathrm{~T}_{\mathrm{c} 2=} & 317.5-0.71 \mathrm{P}-0.96 \mathrm{~L} / \mathrm{D}-5.33 \mathrm{~N}+0.112 \mathrm{P}^{*} \mathrm{P} \\
& +0.0484 \mathrm{~L} / \mathrm{D}^{*} \mathrm{~L} / \mathrm{D}+0.944 \mathrm{~N}^{*} \mathrm{~N} \\
& -0.0417 \mathrm{P}^{*} \mathrm{~L} / \mathrm{D}-0.364 \mathrm{P}^{*} \mathrm{~N}-0.055 \mathrm{~L} / \mathrm{D}^{*} \mathrm{~N} \\
\mathrm{R}^{2}= & 84 \% \quad \\
& \\
\mathrm{COP}_{\mathrm{h} 1}= & -0.0832+0.01032 \mathrm{P}+0.00236 \mathrm{~L} / \mathrm{D} \\
& +0.00951 \mathrm{~N}-0.000539 \mathrm{P}^{*} \mathrm{P} \\
& -0.000071 \mathrm{~L} / \mathrm{D}^{*} \mathrm{~L} / \mathrm{D}-0.00107 \mathrm{~N}^{*} \mathrm{~N} \\
& +0.000035 \mathrm{P}^{*} \mathrm{~L} / \mathrm{D}+0.000138 \mathrm{P}^{*} \mathrm{~N} \\
& -0.000025 \mathrm{~L} / \mathrm{D}^{*} \mathrm{~N} \quad \mathrm{R}^{2}=91.1 \%
\end{aligned}
$$

$$
\begin{aligned}
\mathrm{COP}_{\mathrm{c} 1}= & -0.099+0.015 \mathrm{P}+0.0025 \mathrm{~L} / \mathrm{D} \\
& +0.00636 \mathrm{~N}-0.000706 \mathrm{P}^{*} \mathrm{P} \\
& -0.000056 \mathrm{~L} / \mathrm{D}^{*} \mathrm{~L} / \mathrm{D}-0.00916 \mathrm{~N}^{*} \mathrm{~N} \\
& -0.000041 \mathrm{P}^{*} \mathrm{~L} / \mathrm{D}+0.000254 \mathrm{P}^{*} \mathrm{~N} \\
& -0.000035 \mathrm{~L} / \mathrm{D}^{*} \mathrm{~N} \quad \mathrm{R}^{2}=94.8 \%
\end{aligned}
$$$$
\mathrm{COP}_{\mathrm{h} 2}=0.013+0.00339 \mathrm{P}+0.00025 \mathrm{~L} / \mathrm{D}
$$$$
-0.00403 \mathrm{~N}-0.000245 \mathrm{P}^{*} \mathrm{P}
$$$$
-0.000048 \mathrm{~L} / \mathrm{D}^{*} \mathrm{~L} / \mathrm{D}-0.000161 \mathrm{~N}^{*} \mathrm{~N}
$$$$
+0.000044 \mathrm{P}^{*} \mathrm{~L} / \mathrm{D}+0.000125 \mathrm{P}^{*} \mathrm{~N}
$$$$
+0.0000162 \mathrm{~L} / \mathrm{D}^{*} \mathrm{~N} \quad \mathrm{R}^{2}=86.9 .1 \%
$$

$$
\begin{aligned}
\mathrm{COP}_{\mathrm{c} 2}= & -0.208+0.0215 \mathrm{P}+0.0132 \mathrm{~L} / \mathrm{D} \\
& +0.0356 \mathrm{~N}-0.00169 \mathrm{P}^{*} \mathrm{P} \\
& -0.00042 \mathrm{~L} / \mathrm{D}^{*} \mathrm{~L} / \mathrm{D}-0.0068 \mathrm{~N}^{*} \mathrm{~N} \\
& +0.000176 \mathrm{P}^{*} \mathrm{~L} / \mathrm{D}+0.00327 \mathrm{P}^{*} \mathrm{~N} \\
& +0.00004 \mathrm{~L} / \mathrm{D}^{*} \mathrm{~N} \quad \mathrm{R}^{2}=84.8 \%
\end{aligned}
$$

In the above Figure 4 , the maximum $T_{h 1}$ value has obtained as $310.5 \mathrm{~K}\left(37.5^{\circ} \mathrm{C}\right)$ at experimental run 11 . At this run the set of input variables are pressure $(P)=10$ bar, 
number of nozzles $(N)=5$ and length $(L) /$ diameter $(D)=10$.

The lowest value of $\mathrm{T}_{c 1}$ has obtained in Figure 5 as $294.9 \mathrm{~K}\left(21.9^{\circ} \mathrm{C}\right)$ at experimental test run 11 , the set of input variables are $P=10$ bar, $N=5$ and $L / D$ ratio $=10$.

Figure 6 trend observation has revealed that the maximum $\mathrm{T}_{h 2}$ value $327.1 \mathrm{~K}\left(54.1^{\circ} \mathrm{C}\right)$ has obtained at experimental run 18. At this run the set of input variables are $P=12$ bar, $N=5$ and $L / D$ ratio $=20$. The increase in $\mathrm{T}_{\mathrm{h} 2}$ value at stage 2 in the Vortex tube is due to the variation in pressure and $L / D$ ratio.

The lowest $T c_{2}$ value $284.2 \mathrm{~K}\left(11.2^{\circ} \mathrm{C}\right)$ in the above Figure 7 has observed at experimental run 11 is because of controlling the highest cold air mass fraction $90 \%$ towards cold stream channel.

Based on the observations from Figure 8, the highest $\mathrm{COP}_{h 1}$ value 0.0188 has observed at Experimental run 11. This because of the maximum mass flow rate and the temperature difference between hot outlet 1 and inlet air.

The maximum $\mathrm{COP}_{c 1}$ value 0.0203 has observed in Figure 9 at experimental run 11 . The chosen $50 \%$ cold mass fraction in the first Vortex tube towards cold outlet 1 helped to decrease temperature. This caused to increase in the temperature difference in stage one Vortex tube and to increase in $\mathrm{COP}_{c 1}$.

Based on the trends observed in Figure 10, the maximum $\mathrm{COP}_{h 2}$ value 0.0179 has found at experimental run 4. At this run the set of input variables are $P=8$ bar, $N=4$ and $L / D$ ratio $=10$. The $\mathrm{COP}_{h 2}$ value was more than $\mathrm{COP}_{h 1}$ because of more temperature difference at stage 2 than stage 1.

The maximum $\mathrm{COP}_{c 2}$ value 0.1628 has observed at experimental run 11 in Figure 11. The $\mathrm{COP}_{c 2}$ value was more compared to COP values at hot outlet 1 , cold outlet 1 , and hot outlet 2. In this case, the chosen $90 \%$ cold mass fraction towards cold outlet 2 helped to increase COP.

\section{Conclusions}

In the present work, using the Taguchi approach with grey relational analysis found the decision making multiresponses optimization of input parameters in a two-stage hot cascade type vortex tube. By using Minitab 18 software, 1st and 2nd order mathematical models were developed for selected input parameters and their multiresponses using experimental data. Based on the analysis, the following statements are concluded.

- In the present optimization, the experimental run 11 has observed an optimal one. At this run, the set of input variables are pressure $10 \mathrm{bar}, L / D$ ratio 15 , and the number of nozzles 5 . The optimal $T_{c 1}$ and $T_{c 2}$ values have found by arranging vortex tubes in a cascade manner for spot cooling applications.

- From the ANOVA table, pressure and L/D ratio $P$-values are less than 0.05 . So these two parameters are the most significant input parameters influencing multi-responses in two-stage hot cascade type vortex tube operation. Another parameter, such as the number of nozzles, does not show any signs of responses.
- The $R^{2}$ value of the 2 nd order model is higher compared to the 1st order model. The higher $R^{2}$ value model represents the best fit of the model. So, the 2 nd order model is best for predicted and experimental results correlation.

- As future work, the first vortex tube cold outlet can also connect as an input of the second vortex tube to get two hot points for spot hot applications.

- In a similar way vortex tubes can also connect multistage hot and cold cascade vortex tube manner as future work.

\section{Nomenclature}

COP Coefficient of Performance

$\mathrm{COP}_{1} \quad$ Coefficient of Performance of Cold Outlet 1

$\mathrm{COP}_{1}$ Coefficient of Performance of Hot Outlet 1

$\mathrm{COP}_{2}$ Coefficient of Performance of Cold Outlet 2

$\mathrm{COPh}_{2}$ Coefficient of Performance of Hot Outlet 2

$D \quad$ Hot tube internal diameter

$\xi \quad$ Identification coefficient

$L \quad$ Hot tube length

$N \quad$ Number of Nozzles

$T_{h 1} \quad$ Hot Outlet 1 air temperature $(\mathrm{K})$

$T_{c 1} \quad$ Cold Outlet 1 air temperature (K)

$T_{h 2} \quad$ Hot Outlet 2 air temperature $(\mathrm{K})$

$T_{c 2} \quad$ Cold Outlet 2 air temperature $(\mathrm{K})$

$P \quad$ Air pressure at the inlet (bar)

\section{References}

1. Y.T. Wua, Y. Dinga, Y.B. Jia, C.F. Maa, M.C. Ge, Modification and experimental research on vortex tube, Int. J. Refrigerat. 30, 1042-1049 (2007)

2. K. Dincer, S. Baskaya, B.Z. Uysal, Experimental Investigation of the effects of length to diameter ratio and nozzle number on the performance of counter flow RanqueHilsch vortex tubes, Heat Mass Transfer 44, 367-373 (2008)

3. A. Pinar, M.O. Uluer, V. Kirmaci, Optimization of counter flow Ranque-Hilsch vortex tube performance using Taguchi method, Int. J. Refrigerat. 32, 1487-1494 (2009)

4. O. Aydın, B. Markal, M. Avcı, A new vortex generator geometry for a counter-flow Ranque-Hilsch vortex tube, Appl. Thermal Eng. 30, 2505-2511 (2010)

5. K. Dincer, Experimental investigation of the effects of three-fold type Ranque-Hilsch vortex tube and six cascade type Ranque-Hilsch vortex tube on the performance of counter flow Ranque-Hilsch vortex tubes, Int. J. Refriger. 34, 1366-1371 (2011)

6. J. Prabakaran, S. Vaidyanathan, D. Kanagarajan, Establishing empirical relation to predict temperature difference of vortex tube using response surface methodology, J. Eng. Sci. Technol. 7, 722-731 (2012)

7. A. Berber, K. Dincer, Y. Yılmaz, D.N. Ozen, Rule-based Mamdani-type fuzzy modeling of heating and cooling performances of counter-flow Ranque-Hilsch vortex tubes with different geometric construction for steel, Energy J. 51, 297-304 (2013) 
8. M. Bovand, M.S. Valipour, K. Dincer, S. Eiamsa-ard, Application of response surface methodology to optimization of a standard Ranque-Hilsch vortex tube refrigerator, Appl. Thermal Eng. 67, 545-553 (2014)

9. P. Jayaraman, L. Mahesh Kumar, Multi-response optimization of machining parameters of turning AA6063 T6 aluminum alloy using grey relational analysis in Taguchi method, Sci. Direct Proc. Eng. 97, 197-204 (2014)

10. D. Venkata Sivareddy, P. Vamsi Krishna, A. Venu Gopal, C. L. Prithvi Raz, Parameter optimization in vibration assisted turning of Ti6Al4V alloy using ANOVA and grey relational analysis, Int. J. Autom. Mech. Eng. 15, 5400-5420 (2018)

11. V.H. Nguyen, T.N. Huynh, T.P. Nguyen, T.T. Tran, Single and multi-objective optimization of processing parameters for fused deposition modelling in 3D printing technology, Int. J. Autom. Mech. Eng. 17, 7542-7551 (2020)

12. A.F. Hawary, M.I. Ramdan, Hybrid hydraulic vehicle parameter optimization using multi-objective genetic algorithm, Int. J. Autom. Mech. Eng. 16, 7007-7018 (2019)

13. K.G. Hellyar, Gas Liquefaction using a Ranque-Hilsch Vortex Tube: Design Criteria and Bibliography. Report for the Degree of Chemical Engineer (Massachusetts Institute of Technology; 1979)

14. N. Logothetis, A. Haigh, Characterizing and optimizing multi-response by Taguchi method, Qual. Reliability 4, 159$169(1988)$

15. J.L. Deng, Introduction to Grey system, J. Grey Syst. 1, 1-24 (1989)
16. G. Taguchi, Introduction to Quality Engineering (Asian Productivity Organization, Tokyo, 1990)

17. G.S. Peace, Taguchi Method (Addison-Wesley, 1993)

18. P.J. Ross, Taguchi Techniques for Quality Engineering, 2nd edn. (McGraw-Hill, New York, 1996)

19. K. Dincer, S. Tasdemir, S. Baskaya, B.Z. Uysal, Modeling of the effects of length to diameter ratio and nozzle number on the performance of counter flow Ranque-Hilsch vortex tubes using artificial neural networks, Appl. Thermal Eng. 28, 2380-2390 (2008)

20. A.M. Pinar, O. Uluer, V. Kirmaci, Optimization of counterflow Ranque-Hilsch Vortex Tube performance using Taguchi method, Int. J. Refrigerat. 32, 1487-1494 (2009)

21. S. Eiamsa-ard, K. Wongcharee, P. Promvonge, Experimental investigation on energy separation in a counter-flow RanqueHilsch vortex tube: effect of cooling a hot tube, Int. Commun. Heat Mass Transfer 37, 156-162 (2010)

22. M. Bovand, M.S. Valipour, K. Dincer, A. Tamayol, Numerical analysis of the curvature effects on Ranque-Hilsch vortex tube refrigerators, Appl. Thermal Eng. 65, 176-183 (2014)

23. M. Bovand, M.S. Valipour, S. Eiamsa-ard, A. Tamayol, Numerical analysis for curved vortex tube optimization, Int. Commun. Heat Mass Transfer 50, 98-107 (2014)

24. V. Kirmaci, Experimental investigation of cooling - heating performance of counter flow Ranque-Hilsch vortex tubes having different length diameter ratio, Cumhuriyet Sci. J. 38, 813-821 (2017)

Cite this article as: Racharla Madhu Kumar, NVVS Sudheer, Katam Ganesh Babu, Multi-attribute decision making parametric optimization in two-stage hot cascade vortex tube through grey relational analysis, Int. J. Simul. Multidisci. Des. Optim. 11, 21 $(2020)$ 